\title{
Surface hardening by means of plasma immersion ion implantation and nitriding in glow discharge with electrostatic confinement of electrons
}

\author{
Sergey N. Grigoriev, Alexander S. Metel, Marina A. Volosova \\ AND YURY A. MELNIK ${ }^{\mathrm{a}}$ \\ Moscow State University of Technology "STANKIN", 1 Vadkovsky per., 127994 Moscow GSP-4, Russian Federation
}

Received 27 September 2015, Accepted 19 October 2015

\begin{abstract}
At argon or nitrogen pressure 0.005-5 $\mathrm{Pa}$ a metal substrate is immersed in plasma of glow discharge filling a vacuum chamber and negatively biased to $U=1-10 \mathrm{kV}$. Emitted by substrate secondary electrons bombard the chamber walls and it results in growth of electron emission chamber walls and rise of gas ionization intensity inside chamber. At pressure $p<0.05 \mathrm{~Pa}$ nonuniformity of plasma density does not exceed $\sim 10 \%$. Measurement results of sheath width $d$ between homogeneous plasma and a lengthy flat substrate were used to calculate dependence of the ion current density $j_{i}$ on its surface and ionelectron emission coefficient $\gamma_{\mathrm{i}}$. The dependence allows evaluation of average dose of ion implantation on any complex-shaped product of the same material.
\end{abstract}

Key words: Surface hardening / ion implantation / plasma / glow discharge / nitriding

\section{Introduction}

Plasma immersion ion implantation (PIII) is nowadays widely used in industry for surface strengthening of various machine parts. Essence of this method consists in immersion of product to be treated in plasma and application to this product of negative high-voltage pulses, which extract from plasma and accelerate ions $[1,2]$. Width of surface layer modified through ion implantation is determined by depth of penetration into material of ions bombarding its surface. Depth of ion penetration is directly proportional to energy of ions, and for this reason width of modified layer reaches maximal at applied voltage value only if ions pass through positive space charge sheath between plasma and product surface without collisions with gas molecules.

At voltage of tens kilovolts sheath width $d$ may amount to tenths of one meter. In order to treat any product it is needed to fill with homogeneous plasma a chamber with sizes exceeding the sum of product width and doubled sheath width. Despite sophisticated methods of plasma production, expensive microwave power supplies and other equipment used to fill vacuum chamber with homogeneous plasma, working nitrogen pressure in vacuum chambers of known facilities.

${ }^{a}$ Corresponding author: yu_melnik@mail.ru
It was shown that using glow discharge with electrostatic confinement of electrons [3] any vacuum chamber may be filled with dense and quite homogeneous plasma at argon pressure $0.005-0.05 \mathrm{~Pa}$. The present work describes peculiarities of this discharge, when it is used for plasma immersion ion implantation.

\section{Experimental setup}

Figure 1 presents schematic of the experimental setup. Into 0.5-m-diameter chamber 1 with inner surface area $S=1.46 \mathrm{~m}^{2}$ using feedthrough 2 on bottom flange 3 is introduced flat rectangular anode 4 . Second feedthrough 5 on upper flange 6 is used to fasten inside chamber 1 a product to be treated, for instance, 0.25-m-high and $0.44-\mathrm{m}$-long flat substrate 7 . Chamber 1 is pumped by diffusion pump down to residual gas pressure $p \sim 3 \times$ $10^{-3} \mathrm{~Pa}$. Channel between chamber 1 and diffusion pump 8 is crossed by grid 9 made of 2 -mm-thick titanium sheet perforated with uniformly dispersed 4-mm-diameter holes at distance between hole centers amounting to $5 \mathrm{~mm}$.

Gas pressure is measured by two vacuummeter VIT-3 with ion gauges PMI-2 and PMI-51 (not shown in Fig. 1) and regulated in the range $0.005-5 \mathrm{~Pa}$ using needle valve 10. There is on chamber door a 120 -mm-diameter quartz window 11 used to measure with pyrometer temperature of products under treatment as well as width of sheath 12 


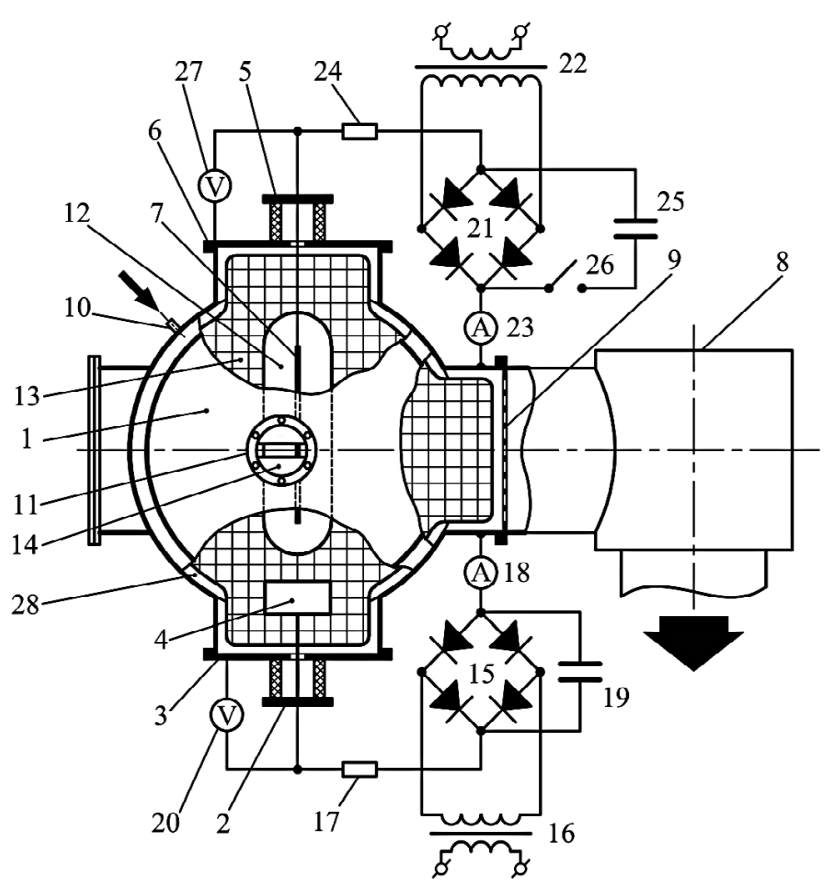

Fig. 1. Experimental setup: (1) vacuum chamber, $(2,5)$ feedthroughs, $(3,6)$ flanges, (4) anode, (7) product, (8) diffusion pump, (9) grid, (10) needle valve, (11) window, (12, 28) sheaths of positive space charge, (13) plasma, (14) slit diaphragm, $(15,21)$ rectifiers, $(16,22)$ transformers, $(17,24)$ resistors, $(18,23)$ ammeters, $(19,25)$ capacitors, $(20)$ voltmeter, (26) switch, (27) kilovoltmeter.

between plasma 13 and flat substrate 7 . Inner surface of window 11 is covered by grounded diaphragm 14 with perpendicular to substrate 7, 2-mm-wide slit. Upper border of the slit has millimetric scale used to register coordinate of flat substrate surface and coordinate of boundary between sheath 12 and plasma 13. Both coordinates are being registered, when line of sight is in plane of substrate surface or in plane of plasma boundary. Sheath width is evaluated as difference of above coordinates.

Rectifier 15 of booster transformer 16 with voltage regulation of its primary winding is used as discharge power supply. Positive pole of rectifier 15 is connected to anode 4 through resistor 17 , whose resistance may be varied from $125 \Omega$ up to $1 \mathrm{k} \Omega$. Its negative pole is connected to chamber 1 through ammeter 18 and a current-measuring shunt (not shown in Fig. 1). Capacitance of filtering capacitor 19 amounts to $80 \mu \mathrm{F}$. Discharge voltage between anode 4 and chamber 1 is measured by voltmeter 20 .

Rectifier 21 of booster transformer 22 with voltage regulation of its primary winding is used as power supply of voltage between substrate 7 and chamber 1 . Its positive pole is connected to chamber 1 through ammeter 23 and a current-measuring shunt (not shown in Fig. 1) and negative pole is connected to substrate 7 through resistor 24 with $3-\mathrm{k} \Omega$ resistance. Filtering condenser 25 with capacitance of $40 \mu \mathrm{F}$ may be connected to rectifier 21 or disconnected from it using switch 26 . Voltage between chamber 1 and substrate 7 is measured by kilovoltmeter

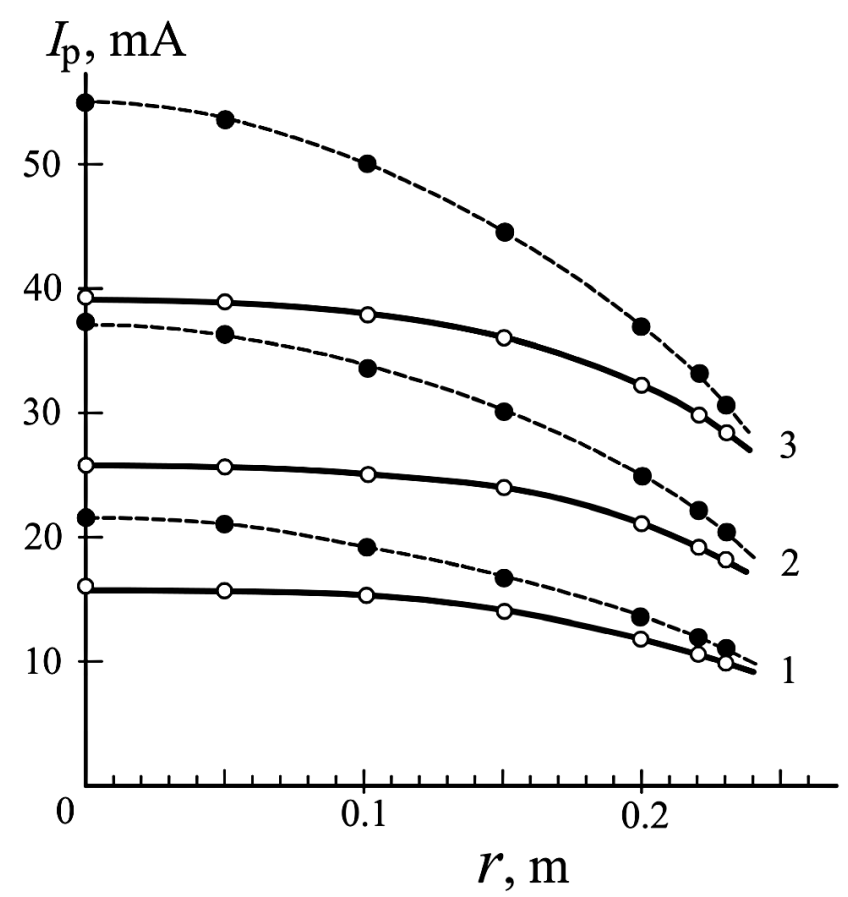

Fig. 2. Dependence of current $I_{\mathrm{p}}$ in probe circuit on distance $r$ between probe and chamber axis at nitrogen pressure $0.05 \mathrm{~Pa}$ (full curves) and $0.5 \mathrm{~Pa}$ (dashed curves). Anode surface area $S_{\mathrm{a}}=0.32 \mathrm{~m}^{2}$, discharge current $I_{\mathrm{d}}=0.5$ (curve 1), 1 (2) and 2 A (3).

27 as well as using oscilloscope traces of signal waveform from connected between them voltage divider (not shown in Fig. 1). When switch 26 is opened currents in circuits of anode 4 and substrate 7 may be measured using oscilloscope traces of signal waveform from above shunts.

\section{Experimental results}

At a voltage $\sim 1 \mathrm{kV}$ between anode 4 and chamber 1 increase of pressure up to $\sim 1 \mathrm{~Pa}$ results in breakdown and initiation of gas discharge. After breakdown discharge voltage amounts to $U_{\mathrm{d}} \sim 500 \mathrm{~V}$, and chamber is filled with bright and quite homogeneous glow of plasma 13, which is separated from chamber walls by cathode sheath 28 and from product 7 to be treated by sheath 12. Multiplication of fast electrons in the cathode sheath play a leading role in the discharge sustaining. Glow intensity inside sheaths is substantially lower than in plasma. At discharge current $I_{\mathrm{d}}=2 \mathrm{~A}$ in circuit of anode 4 and voltage $U_{\mathrm{d}} \approx 500 \mathrm{~V}$ between anode and chamber 1 mean width of cathode sheath exceeds radius of grid 9 holes several times. For this reason within the whole range of investigated parameters plasma 13 does not penetrate into diffusion pump.

Figure 2 presents dependence of current $I_{\mathrm{p}}$ in circuit of $100-\mathrm{mm}$-diameter disc probe, which is electrically connected to chamber, on distance $r$ between probe and chamber axis at anode surface area $S_{\mathrm{a}}=0.032 \mathrm{~m}^{2}$ and discharge current $I_{\mathrm{d}}$ ranging from 0.5 to $2 \mathrm{~A}$. It indicates 


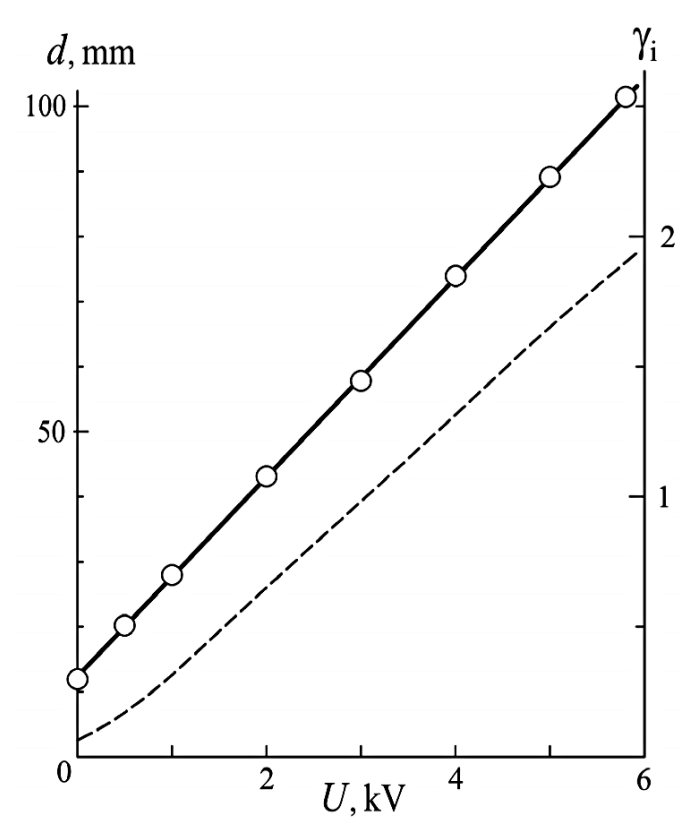

(a)

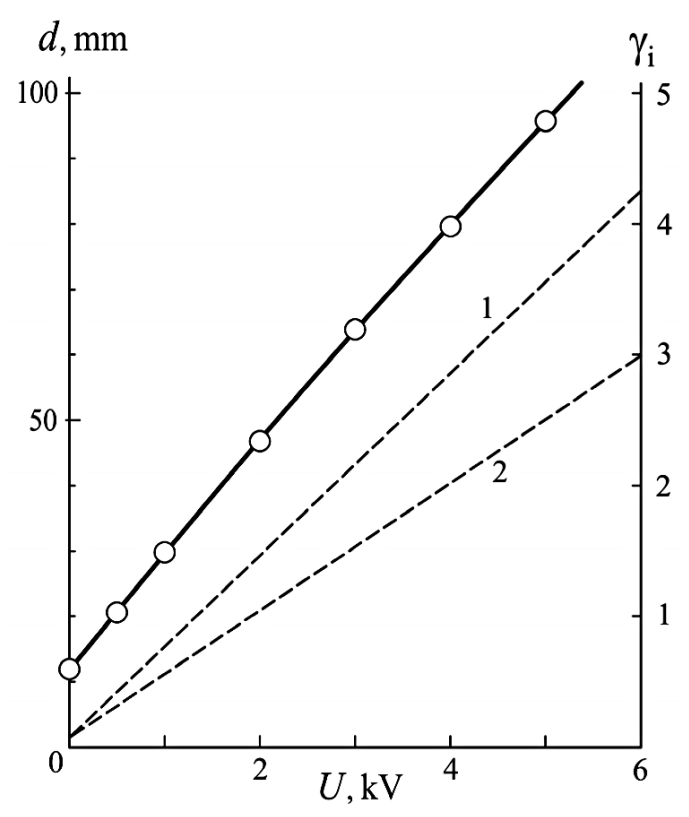

(b)

Fig. 3. Dependence of sheath width $d$ between plasma and flat titanium substrate on voltage $U$ between chamber and substrate at argon (a) and nitrogen (b) pressure $p=0.04 \mathrm{~Pa}$ (full curves) as well as calculated dependence on $U$ of secondary ion-electron emission coefficient $\gamma_{\mathrm{i}}$ (dashed curves) for plasma comprising only molecular nitrogen ions (curve 1) or only atomic nitrogen ions (2) (b).

that nitrogen plasma nonuniformity does not exceed $20 \%$ at pressure $p \sim 0.5 \mathrm{~Pa}$ and $10 \%$ at $p \leq 0.5 \mathrm{~Pa}$.

Placement in the chamber center of substrate 7 made of 2-mm-thick titanium sheet results in increase of discharge cathode working area $1.46 \mathrm{~m}^{2}$ by substrate surface area $0.22 \mathrm{~m}^{2}$, but it does not increase nonuniformity of plasma density distribution inside chamber. When voltage $U$ between chamber 1 and substrate 7 is equal to zero, width of sheath 12 (Fig. 1) between plasma 13 and substrate 7 is practically equal to width of sheath 28 between plasma 13 and chamber 1 . However after switch 26 is closed and transformer 22 is powered on increase of voltage $U$ between chamber and substrate at constant discharge current $I_{\mathrm{d}}$ is accompanied by substantial widening of sheath 12 and growth of current $I$ in substrate circuit. Moreover voltage $U_{\mathrm{d}}$ between anode and chamber diminishes several times.

Full curve in Figure 3a presents experimental dependence of sheath width $d$ between plasma 13 and flat substrate 7 (Fig. 1) on voltage $U$ between chamber and substrate at constant discharge current $I_{\mathrm{d}}=0.5 \mathrm{~A}$ and argon pressure 0.04 Pa.

As at this pressure maximal measured value $d \sim 0.1 \mathrm{~m}$ is shorter than charge exchange length $\lambda_{\mathrm{c}} \approx 0.4 \mathrm{~m}$ of argon ions, dependence of ion current density $j_{\mathrm{i}}$ on sheath width $d$ and potential difference between plasma and substrate, which amounts to $\left(U+U_{\mathrm{d}}\right)$, obeys the Child-Langmuir law. After we measured current $I$ in substrate circuit and using the Child-Langmuir law calculated ion current $I_{\mathrm{i}}$, it is easy to determine current of secondary electrons $I_{\mathrm{e}}=I-I_{\mathrm{i}}$ and coefficient of secondary ion-electron emission $\gamma_{\mathrm{i}}=I_{\mathrm{e}} / I_{\mathrm{i}}$. Dashed curve in Figure 3 a presents calculated in this way dependence of $\gamma_{\mathrm{i}}$ on bias voltage $U$ of a titanium substrate.

Full curve in Figure 3b presents experimental dependence $d(U)$ in nitrogen plasma at discharge current $I_{\mathrm{d}}=0.8 \mathrm{~A}$ and gas pressure $0.04 \mathrm{~Pa}$. As at this pressure charge exchange length $\lambda_{\mathrm{c}} \approx 0.4 \mathrm{~m}$ of molecular nitrogen ions also exceeds $d$, presented in Figure $3 \mathrm{~b}$ by dashed curve 1 dependence of $\gamma_{i}$ on $U$ for these ions was determined in the same way. In addition to molecular ions there are in nitrogen plasma atomic ions. Dashed curve 2 in Figure 3 b presents dependence of $\gamma_{i}$ on $U$ calculated for atomic nitrogen ions. True value of $\gamma_{\mathrm{i}}$ depends on ratio of atomic ion current to molecular ion current in circuit of product under treatment in nitrogen plasma and lies between dashed curves 1 and 2 . For instance, in order to estimate rate of nitrogen implantation dose rise $v$ at $I_{\mathrm{d}}=2 \mathrm{~A}, U=6 \mathrm{kV}$ and $I=1.1$ A we may suppose that $\gamma_{\mathrm{i}} \approx(4.2+3) / 2=3.6$ (see Fig. 3b). In this case ion current in the product circuit $I_{\mathrm{i}}=I /\left(1+\gamma_{\mathrm{i}}\right)=1.1 /(1+3.6)=0.24$ A. To evaluate average ion current density $j_{\mathrm{i}}$ we divide $I_{\mathrm{i}}$ by product surface area and to evaluate average rate of implantation dose rise $v$ we have to divide $j_{\mathrm{i}}$ by electron charge $e$. It is known that implantation dose density resulting in maximal effect of surface modification ranges from $10^{20}$ up to $10^{22} \mathrm{~m}^{-2}$. In our case it corresponds to treatment time ranging from $15 \mathrm{~s}$ up to $25 \mathrm{~min}$, which is substantially shorter than using plasma immersion ion implantation systems equipped with generators of highvoltage pulses. It should be mentioned that any increase 


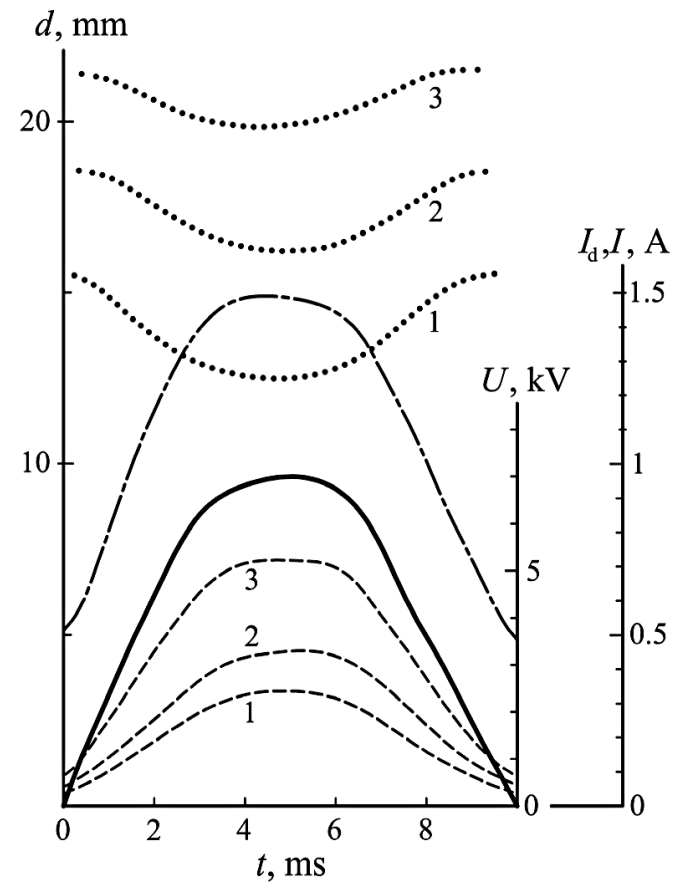

(a)

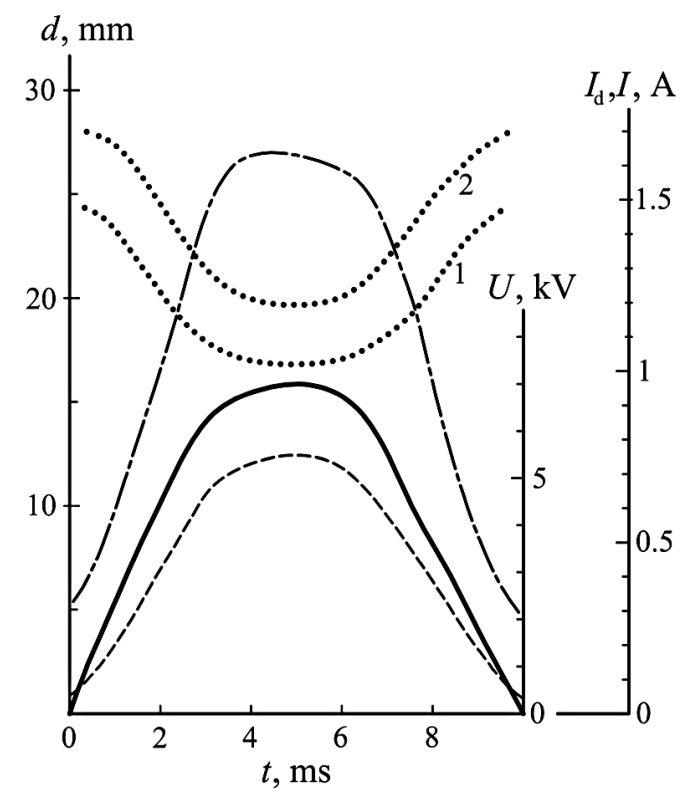

(b)

Fig. 4. Oscillograms of voltage $U$ between chamber and flat titanium substrate (full curve), current $I_{\mathrm{d}}$ in anode circuit (dashdotted curve) and current $I$ in circuit of the substrate (dashed curves) with area $S_{\mathrm{s}}=0.03$ (curve 1$), 0.07(2)$ and $0.17 \mathrm{~m}^{2}(3)$ at opened switch 26 (Fig. 1), argon pressure $0.09 \mathrm{~Pa}$ and averaged effective values of oscillating voltages and currents $U=5 \mathrm{kV}$, $I_{\mathrm{d}}=1 \mathrm{~A}, I=0.2(1), 0.3(2), 0.5 \mathrm{~A}(3)$ and $U_{\mathrm{d}}=380(1), 250(2), 220 \mathrm{~V}(3)$ as well as dependence on time $t$ of sheath width $d$ between plasma and substrate (dotted curves) (a); oscillograms of $U$ (full curve), $I_{\mathrm{d}}$ (dash-dotted curve) and current $I$ in circuit of 0.2-m-diameter and 0.115-m-long hollow titanium cylinder (dashed curve) at nitrogen pressure 0.09 Pa and effective voltages and currents $U=5 \mathrm{kV}, I_{\mathrm{d}}=1 \mathrm{~A}, I=0.5 \mathrm{~A}$ and $U_{\mathrm{d}}=250 \mathrm{~V}$ as well as dependence of sheath width $d$ between cylinder and plasma on time $t$ (dotted curves) calculated for molecular (1) and atomic (2) nitrogen ions.

of implantation rate $v$ results in temperature rise of product under treatment. As to the above example, power density of product heating by ions amounts to $j_{\mathrm{i}}\left(U+U_{\mathrm{d}}\right)=$ $(0.24 / 0.22) \times(6000+200)=6.8 \times 10^{3} \mathrm{VA} \cdot \mathrm{m}^{-2}$ and is equal to radiance of titanium product with emissivity 0.25 at temperature $560{ }^{\circ} \mathrm{C}$, which is sufficient, for instance, for effective high-speed steel nitriding. During treatment product temperature may be regulated by means of voltage $U$ and discharge current $I_{\mathrm{d}}$ variation, the latter being proportional to current $I_{\mathrm{i}}$ of bombarding ions.

It is impossible at high value of sheath width $d$ to treat surface of products with complex shape, for instance, inner surface of 0.2 -m-diameter hollow cylinder, when at $d>0.1 \mathrm{~m}$ plasma does not penetrate into the cylinder. In order to reduce $d$ we should decrease $U$ and energy of ions, which would result in undesirable width reduction of modified through implantation layer, or should increase ion current density on product surface, which at used in this work simplest electrical scheme would lead to rise of power dissipated in resistor 24 (Fig. 1) preventing cathode spots of vacuum arc from appearing on product under treatment. Nevertheless at the same effective values of high voltage $U$ and current $I$ in product circuit sheath width $d$ was appreciably reduced after filtering of rectified voltage $U$ was abandoned. Figure 4a presents oscillograms of voltage $U$, current $I$ in circuit of flat substrate with surface area $S_{\mathrm{s}}=0.03(1), 0.07(2), 0.17 \mathrm{~m}^{2}(3)$ and discharge current $I_{\mathrm{d}}$ in anode circuit at opened switch 26 (Fig. 1), argon pressure $0.09 \mathrm{~Pa}$, resistance $125 \Omega$ of resistor 17 and measured with voltmeters and ammeters averaged effective values of oscillating voltages and currents $U=5 \mathrm{kV}$, $I_{\mathrm{d}}=1 \mathrm{~A}, I=0.2(1), 0.3(2), 0.5 \mathrm{~A}(3)$ and $U_{\mathrm{d}}=380$ (1), $250(2), 220 \mathrm{~V}(3)$.

Current $I$ in substrate circuit rises with its surface area $S_{\mathrm{s}}$, but discharge voltage $U_{\mathrm{d}}$ diminishes. Dotted curves in Figure 4a present dependence on time $t$ of sheath width $d$ calculated with the use of oscillograms $I(t), U(t)$, dependence $\gamma_{\mathrm{i}}(U)$ and Child-Langmuir law (2). Sheath width $d$ ranges from $13 \mathrm{~mm}$ to $22 \mathrm{~mm}$, which is substantially lower than at closed switch 26 and the same effective parameters $U, I_{\mathrm{d}}, I, U_{\mathrm{d}}$. When potential difference $\left(U+U_{\mathrm{d}}\right)$ between plasma and substrate rises from $\sim 0.5 \mathrm{kV}$ at $t=0$ up to $\sim 7.5 \mathrm{kV}$ at $t=5 \mathrm{~ms}$, current in substrate circuit grows up by an order of magnitude. For this reason sheath width $d$ does not increase, but on the contrary diminishes by $10-20 \%$. When substrate surface area $S_{\mathrm{s}}$ rises from 0.03 to $0.17 \mathrm{~m}^{2}$, at invariant pulse of current $I_{\mathrm{d}}$ in anode circuit amplitude of current $I$ in substrate circuit grows up, but sheath width $d$ at $t=5 \mathrm{~ms}$ also rises from 13 up to $20 \mathrm{~mm}$. 
Figure $4 \mathrm{~b}$ presents oscillograms of discharge current $I_{\mathrm{d}}$, voltage $U$ and current $I$ in circuit of product shaped as 0.2 -m-diameter and 0.115 -m-long hollow cylinder made of 2-mm-thick titanium sheet at nitrogen pressure 0.09 Pa and averaged effective values $U=5 \mathrm{kV}, I_{\mathrm{d}}=1 \mathrm{~A}, I=$ $0.5 \mathrm{~A}$ and $U_{\mathrm{d}}=250 \mathrm{~V}$. Dotted curves present calculated dependence of sheath width $d$ on timetfor plasma containing only molecular nitrogen ions (curve 1) or atomic nitrogen ions (curve 2). In both cases $d$ does not exceed $28 \mathrm{~mm}$, for this reason plasma freely penetrates into hollow cylinder, and ions accelerated in sheath between plasma and cylinder inner surface bombard this surface quite uniformly.

This was confirmed by treatment of positioned on cylinder inner surface cutting plates made of high-speed steel at nitrogen pressure $0.09 \mathrm{~Pa}$, voltage $U=6 \mathrm{kV}$ and anode current $I_{\mathrm{d}} \sim 2 \mathrm{~A}$. Two plates were positioned on brinks of the inner surface and the third plate was positioned just in the middle of them. After 1-hour-long treatment at temperature $500{ }^{\circ} \mathrm{C}$ of outer cylinder surface, which was measured with pyrometer and regulated through variation of effective discharge current $I_{\mathrm{d}}$, microhardness of all three plates increased from $950 \mathrm{HV}$ up to $1400 \mathrm{HV}$ at $25 \mathrm{-g}$ indenter load. However increase of the load up to $50 \mathrm{~g}$ results in decrease of measuring result down to $1300 \mathrm{HV}$. Dependence of measuring result on the load may be explained by higher hardness of modified by nitrogen implantation surface layer with thickness not exceeding $0.1 \mu \mathrm{m}$, from which nitrogen propagates further into diffusion saturation layer with thickness up to $50 \mu \mathrm{m}$.

Characterization of treated plates showed additionally that bombardment of high-speed steel by $6-\mathrm{keV}$ ions prevents from production on its surface of iron nitride film. At ion energy of several hundreds electronvolts and nitrogen pressure exceeding $1 \mathrm{~Pa}$ this film is always present on steel surface and reduces the nitriding rate. In order to diminish its influence and increase the nitriding rate gas mixture of nitrogen with argon or helium is used. In our case of nitriding with high-energy ions it is possible to abandon any additions.

When material heating over some critical temperature $T_{\mathrm{o}}$ being lower than temperature of effective thermodiffusion is not acceptable, material surface strengthening should be limited to ion implantation. In order that product temperature does not exceed $T_{\mathrm{o}}$, high voltage power supply may be periodically switched off for a time ensuring maintenance of controlled by pyrometer working temperature $T<T_{\mathrm{o}}$ and then switched on again. In this case treatment time may exceed mentioned above value ranging from $15 \mathrm{~s}$ up to $25 \mathrm{~min}$, but in any case it would be lower than for plasma immersion ion implantation using high-voltage pulse generator.

It is needed to emphasize that the above method can be applied only to products made of conductive materials. For processing of dielectric materials can be used broad beam sources of ions [4-6] or fast neutral molecules [7-12]. The sources allow nitriding of products without blunting their sharp edges. Beams of fast neutral molecules can be also produced without sources through immersion of high-transparency grids in the discharge plasma filling the working chamber [7].

Nevertheless, strengthening the surface of conductive products through the above plasma immersion ion implantation method [13] seems to be more applicable to industry then even methods of wear-resistant coating deposition [14].

\section{Conclusions}

1. At pressure $0.005-5 \mathrm{~Pa}$ any vacuum chamber with volume $0.1-1 \mathrm{~m}^{3}$ may be filled using glow discharge with electrostatic confinement of electrons not only with plasma of inert gases, but also with reactive plasma its nonuniformity not exceeding 10-20\%.

2. Bombardment of chamber walls by fast electrons emitted by immersed in plasma and negatively biased product raises by an order of magnitude current of emitted by chamber electrons and intensity of gas ionization inside chamber. As a result cathode fall of potential diminishes from hundreds to tens of volts, and intensity of chamber sputtering by ions falls down to zero.

3. During nitrogen ion implantation rate of dose rise at constant high voltage $U$ applied to the product under treatment is by an order of magnitude higher than in known systems equipped with generators of squarewave high-voltage pulses.

4. When to the product instead of low-ripple rectified high voltage are applied sinusoidal 10-ms-wide highvoltage pulses following each other with $100-\mathrm{Hz}$ frequency, sheath width between plasma and product diminishes several times at the same averaged effective values of high voltage and current in product circuit. This enables to treat products in comparably small vacuum chambers and to treat inner surfaces of hollow products.

Acknowledgements. This work was carried out with the financial support of the Ministry of Education and Science of Russian Federation in the framework of the state task in the field of scientific activity of MSTU "STANKIN" (No. 1059).

\section{References}

[1] A. Metel, Plasma immersion ion implantation based on glow discharge with electrostatic confinement of electrons, Surf. Coat. Technol. 156 (2002) 38-43

[2] A. Anders, Handbook of Plasma Immersion Ion Implantation and Deposition, John Wiley and Sons, New York, 2000

[3] V.I. Kolobov, A.S. Metel, Glow discharges with electrostatic confinement of fast electrons, J. Phys. D 48 (2015) 233001

[4] H.R. Kaufman, Broad-beam ion sources, Rev. Sci. Instrum. 61 (1990) 230-235 
[5] A.V. Hayes, V. Kanarov, B. Vidinsky, Fifty centimeter ion beam source, Rev. Sci. Instrum. 67 (1996) 1638-1641

[6] N.V. Gavrilov, G.A. Mesyats, G.V. Radkovskii, V.V. Bersenev, Development of technological sources of gas ions on the basis of hollow-cathode glow discharges, Surf. Coat. Technol. 96 (1997) 81-88

[7] A.S. Metel, Beams of fast neutral atoms and molecules in low-pressure gas-discharge plasma, Plasma Phys. Rep. 38 (2012) 254-262

[8] E.W. McDaniel, Collision phenomena in ionized gases, John Wiley \& Sons, New York - London - Sydney, 1964

[9] A. Ranjan, V.M. Donnelly, D.J. Economou, Energy distribution and flux of fast neutrals and residual ions extracted from a neutral beam source, J. Vacuum Sci. Technol. A 24 (2006) 1839-1646

[10] A.S. Metel, Yu.A. Melnik, V.V. Panin, Non-self-sustained glow discharge with electrostatic confinement of electrons sustained by a fast neutral molecule beam, Plasma Phys. Rep. 37 (2011) 357-365
[11] A.F. Cakir, A. Metel, M. Urgen, S. Grigoriev, Arc-PVD coating of metallic and dielectric substrates using neutral molecular beam source pretreatment, Galvanotechnik 91 (2000) 768-776

[12] A.S. Metel, S.N. Grigoriev, Yu.A. Melnik, V.P. Bolbukov, Broad beam sources of fast molecules with segmented cold cathodes and emissive grids, Instruments and Experimental Techniques 55 (2012) 122-130

[13] J.C. Avelar-Batista, E. Spain, J. Housden, A. Matthews, G.G. Fuentes, Plasma nitriding of Ti6Al4V alloy and A1S1M2 steel substrates using DC glow discharge under a triode configuration, Surf. Coat. Technol. 200 (2005) 1954-1961

[14] S. Grigoriev, A. Metel, Plasma- and beam-assisted deposition methods, in: Nanostructured thin films and nanodispersion strengthened coatings. Kluwer Academic Publishers, Boston, Dordrecht, London, 2004, pp. $147-154$ 